九州大学学術情報リポジトリ

Kyushu University Institutional Repository

\title{
Investigation of a Laser Scanner for Measurement of Lodging Posture of a Wheat Bunch
}

Hirai, Yasumaru

Department of Bioproduction and Environmental Sciences, Faculty of Agriculture, Kyushu University

Hamagami, Kunihiko

Graduate School of Bioresource and Bioenvironmental Sciences, Kyushu University

Mori, Ken

Department of Bioproduction and Environmental Sciences, Faculty of Agriculture, Kyushu University

https://doi.org/10.5109/10076

出版情報: 九州大学大学院農学研究院紀要. 53 (1)，pp.89-93，2008-02-28. Faculty of Agriculture， Kyushu University

バージョン :

権利関係 : 


\title{
Investigation of a Laser Scanner for Measurement of Lodging Posture of a Wheat Bunch
}

\author{
Yasumaru HIRAI ${ }^{1 *}$, Kunihiko HAMAGAMI ${ }^{2}$ \\ and Ken MORI ${ }^{1}$
}

\begin{abstract}
Laboratory of Bioproduction and Environment Information Sciences, Division of Bioproduction and Environment Information Sciences, Department of Bioproduction and Environmental Sciences, Faculty of Agriculture, Kyushu University, Fukuoka 812-8581, Japan (Received October 17, 2007 and accepted November 30, 2007)
\end{abstract}

\begin{abstract}
A real time sensing technique for detecting lodging posture of crops was investigated using a laser scanner. In the laboratory experiment, posture profiles of a wheat bunch with four lodging directions (left, right, front, back) and three lodging angles $\left(40^{\circ}, 62^{\circ}, 80^{\circ}\right)$ were measured, and the profiles were assessed through comparisons with digital images. Also, inclination, skewness, and kurtosis were calculated using coordinate data of lodging profiles in three dimensions and the effectiveness of those values as indications representing lodging characteristics was assessed. As the results, the inclination of lodging posture was roughly detected for relatively large lodging angles. On the other hand, only upper profiles of a wheat bunch were measured under an upright condition and for the case that an angle of the sensor against a wheat bunch was not arranged appropriately. The limited profiles made it difficult to detect the inclination of lodging posture. Useful information on lodging characteristics, such as a lodging direction and density distribution of a wheat stalk, was obtained from values of skewness and kurtosis under certain conditions while the results were significantly affected by the setting for an angle of the sensor.
\end{abstract}

\section{INTRODUCTION}

In Japan, lodging of crops due to a typhoon often lowers efficiency of harvesting operations. Although a head-feeding type combine used in Japan efficiently harvests lodging crops comparing with a reel header type combine used mainly in Europe and the USA, the operation at a low speed is inevitable to keep the same harvesting accuracy as that under an upright crop condition. Especially, in the case that lodging directions are not uniform, traveling directions during harvesting operations must be adjusted, and the event significantly reduces efficiency. Also, because these adjustments for machine operations depend on skill of each operator, it is hard to say that harvesting operations are optimized under such conditions. Therefore, it is necessary to develop an automatic control system that optimizes cutting and feeding of crops according to lodging conditions, in order to secure stable machine performance. As the first step of the development of such a control system, it is indispensable to detect lodging posture in real time during harvest. Thus, the sensing technique using a laser scanner was investigated.

In recent years, detection of various objects using a laser scanner has been investigated in an agricultural

\footnotetext{
1 Laboratory of Bioproduction and Environment Information Sciences, Division of Bioproduction and Environment Information Sciences, Department of Bioproduction and Environmental Sciences, Faculty of Agriculture, Kyushu University

2 Laboratory of Bioproduction and Environment Information Sciences, Division of Bioproduction and Environment Information Sciences, Department of Bioproduction and Environmental Sciences, Graduate school of Bioresource and Bioenvironmental Sciences, Kyushu University

* Corresponding author (E-mail: hirai@bpes.kyushu-u.ac.jp)
}

field. Monta et al. (2004) used the sensor to capture precise external information needed for operations of an agricultural robot, such as a three dimensional image in a vine yard, shape and the location of a tomato harvested, and the location of human being around a robot. Tumbo et al. (2002) investigated the method for rapid mapping of volume for citrus canopy using a laser sensor, which was necessary for variable rate application of fertilizer and pesticide, and yield estimation. Fox and Wagner (2001) worked on crop residue management related to a wind erosion problem, and used a laser sensor to measure a silhouette area index for standing residue.

The objective of this study was to assess the application of a laser scanner to detection of lodging postures of crops. Lodging posture profiles of a wheat bunch with four lodging directions (left, right, front, back) and three lodging angles $\left(40^{\circ}, 62^{\circ}, 80^{\circ}\right)$ were measured in the laboratory experiment and the profiles were compared with digital images. Also, inclination, skewness, and kurtosis calculated using coordinate data of lodging profiles in three dimensions were evaluated as indications representing lodging characteristics.

\section{EXPERIMENT AND METHOD}

\section{Experimental system}

Lodging profiles of a wheat bunch was measured using the experimental system as shown in Fig.1. The following apparatuses were used in the system: scanning laser range finder (Hokuyo Automatic Co., Ltd, URG-04LX), liner head, DC motor, control unit, 5V DC power supply, notebook computer (Sony, VAIO PCGU3), tripod, criterion bar, and a wheat bunch. The linear head is composed of a rack, a rack case, a rack bush, 
and a reduction gear. Forward and backward motions of the rack were operated by the control unit. The laser scanner was attached to the tip of the rack using a metal fixture that allows direction of an optical axis to be adjusted. The height of the sensor was arranged by the tripod. The laser scanner measures distance from the center of an optical axis to a target object by flight time of emitted light, which is reflected by the target (time of flight principle). Specifications of the sensor are shown in table1. As shown in table 1, distance to a target object is acquired at the angle resolution of $0.36^{\circ}$ within the scanning angle of $240^{\circ}$. Namely, 683 points of distance data are acquired in one scan (100 msec). Distance data was logged on the notebook computer through the USB communication. Then, distance data was transformed into coordinates in three dimensions based on coordinates of the criterion bar.

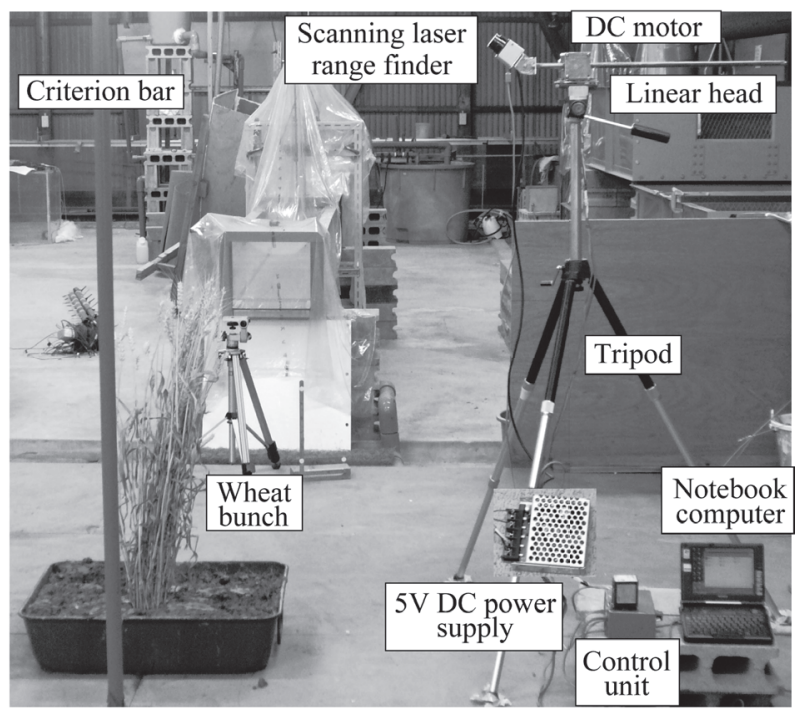

Fig. 1. Experimental system.

Table 1. Specifications of URG-04LX

\begin{tabular}{cc}
\hline Light source & Semiconductor laser, wave length: $785 \mathrm{~nm}$ \\
Power source & $5 \mathrm{VDC}, \pm 5 \%$ \\
Detectable distance & $20 \mathrm{~mm}$ to $4000 \mathrm{~mm}$ \\
Accuracy & Official 20 to $1000 \mathrm{~mm}: \pm 10 \mathrm{~mm}$, \\
Resolution & 1000 to $4000 \mathrm{~mm}: \pm 1 \%$ of measurement \\
Scanning angle & $240^{\circ}$ \\
Angle resolution & Approx. $0.36^{\circ}\left(360^{\circ} / 1024\right.$ steps $)$ \\
Scanning time & $100 \mathrm{msec} / \mathrm{scan}$ \\
Weight & Approx. $160 \mathrm{~g}$ \\
\hline
\end{tabular}

\section{Transformation of distance data into coordinates in three dimensions}

A positional relationship between axes of coordinates, the wheat bunch, and the criterion bar is shown in Fig. 2(a). The $z$ axis in Fig. 2(a) directs upward along the height direction of the wheat bunch. The laser scanner moves forward in the direction of the $y$-axis while distance between the center of an optical axis and the wheat bunch is measured. It results in scanning a partial posture profile of the wheat bunch. Now, since the laser scanner moves during the meas- urement, its current position is determined based on coordinates of the criterion bar. The optical axis is inclined at an angle of $\theta$ against the $y$-axis as shown in Fig. 2(b). Therefore, the rectangular coordinate system $\mathrm{Q}-\xi \eta \zeta$ (moving coordinate system) is introduced. Here, the origin $\mathrm{Q}$ is the center of an optical axis. After coordinates were calculated in this coordinate system $(\mathrm{Q}-\xi \eta \zeta)$, the coordinates were transformed in ones in the moving coordinate system Q-x'y'z' (Fig. 2(c)). The position of the criterion bar at the coordinate system $\mathrm{Q}-\xi \eta \zeta$ is calculated as follows (Fig. 2(d)):

$$
\left\{\begin{array}{l}
\xi_{\mathrm{cri}}=L_{\mathrm{cri}} \cos \alpha_{\mathrm{cri}} \\
\eta_{\mathrm{cri}}=L_{\mathrm{cri}} \sin \alpha_{\mathrm{cri}} \\
\xi_{\mathrm{cri}}=0
\end{array}\right.
$$

where, $L_{\text {cri }}$ : distance from the center of an optical axis to the criterion bar in mm; $\alpha_{\text {cri }}$ : scanning angle of the criterion bar from $\xi$ : axis in degree (positive in the counterclockwise direction); $\xi_{\text {cri }}, \eta_{\text {cri }}, \xi_{\text {cri }}$ : coordinates of the criterion bar at the coordinate system $\mathrm{Q}-\xi \eta \zeta$ in mm. Further, since the coordinate system $\mathrm{Q}-\xi \eta \xi$ rotates through $\theta$ degrees against $y$ '-axis as shown in Fig. 2(c), coordinates of the criterion bar at the coordinate system $\mathrm{Q}-x^{\prime} y^{\prime} z$ ' is calculated from the following relation. Here, the rotational angle $\theta$ is positive in the counterclockwise direction.

$$
\left[\begin{array}{l}
x_{\text {cri }}^{\prime} \\
y_{\text {cri }}^{\prime} \\
z_{\text {cri }}^{\prime}
\end{array}\right]=\left[\begin{array}{ccc}
1 & 0 & 0 \\
0 & \cos \theta & -\sin \theta \\
0 & \sin \theta & \cos \theta
\end{array}\right]\left\{\begin{array}{c}
L_{\text {cri }} \cos \alpha_{\text {cri }} \\
L_{\text {cri }} \sin \alpha_{\text {cri }} \\
0
\end{array}\right\}
$$

where, $x_{\text {cri }}^{\prime}, y_{\text {cri }}^{\prime}, z_{\text {cri }}^{\prime}$ : coordinates of the criterion bar at the coordinate system $\mathrm{Q}-x^{\prime} y^{\prime} z$ ' in $\mathrm{mm}, \theta$ : rotational angle of the laser scanner against $y$ '-axis in degree (positive in the counterclockwise direction). Also, coordinates of an arbitrary object scanned at the coordinate system Q-x'y'z' are calculated from the following relation.

$$
\left[\begin{array}{l}
x^{\prime} \\
y^{\prime} \\
z^{\prime}
\end{array}\right]=\left[\begin{array}{ccc}
1 & 0 & 0 \\
0 & \cos \theta & -\sin \theta \\
0 \sin \theta & \cos \theta
\end{array}\right]\left\{\begin{array}{c}
L \cos \alpha \\
L \sin \alpha \\
0
\end{array}\right\}
$$

where, $L$ : distance from the center of an optical axis to an object in mm; $x^{\prime}, y^{\prime}, z^{\prime}$ : coordinates of an object scanned at the coordinate system $\mathrm{Q}-x^{\prime} y^{\prime} z^{\prime}, \alpha$ : scanning angle of an object from $\xi$ axis in degree (positive in the counterclockwise direction). Also, since the height of the sensor is constant, the current coordinates of the center of an optical axis are calculated from the following equation.

$$
\left\{\begin{array}{l}
X_{\mathrm{L}}=X_{\text {cri }}-x_{\text {cri }}^{\prime} \\
Y_{\mathrm{L}}=Y_{\text {cri }}-y_{\text {cri }}^{\prime} \\
Z_{\mathrm{L}}=\text { const }
\end{array}\right.
$$

where, $X_{\text {cri }}, Y_{\text {cri }}: x, y$ coordinates of the criterion bar at the coordinate system $\mathrm{O}-x y z$ in $\mathrm{mm}, X_{\mathrm{L}}, Y_{\mathrm{L}}, Z_{\mathrm{L}}$ : coordinates of the center of an optical axis at the coordinate system $\mathrm{O}-x y z$ in mm. Now, coordinates of an object at 
the coordinate system $\mathrm{O}-x y z$ is calculated by adding the coordinate of an object seen from the center of an optical axis obtained by equation (3) into the current coordinates of the center of an optical axis obtained by equation (4). Namely, coordinates of an arbitrary object scanned are calculated by the following equation.

$$
\left\{\begin{array}{l}
x=x^{\prime}+X_{\mathrm{L}} \\
y=y^{\prime}+Y_{\mathrm{L}} \\
z=z^{\prime}+Z_{\mathrm{L}}
\end{array}\right.
$$

where: $x, y, z$ : coordinates of an arbitrary object scanned at the coordinate system $\mathrm{O}-x y z$.
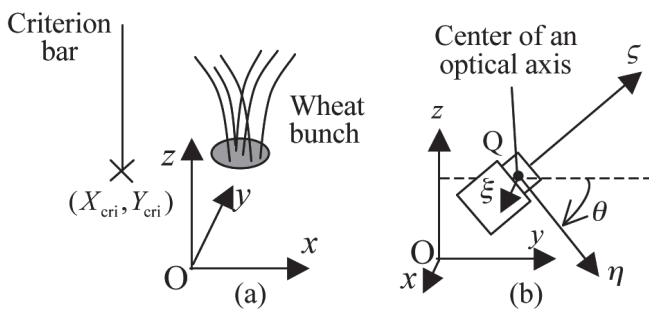

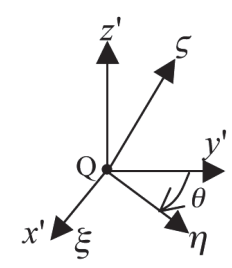

(c)

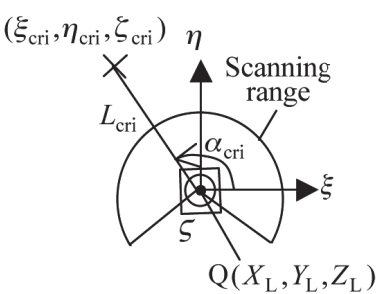

(d)
Fig. 2. Illustrations for transformation of distance data.

\section{Experimental conditions and procedure}

Four lodging directions tested that direct in the axes of positive $x$, negative $x$, negative $y$, and positive $y$ are defined as "right", "left", "front", and "back", respectively. Three lodging angles tested were $40^{\circ}, 62^{\circ}$, and $80^{\circ}$. The same wheat bunch was used throughout the experiment. Digital images of the wheat bunch taken under each experimental condition are shown in Fig. 3, Fig. 4, Fig. 5, and Fig. 6. Four lodging directions were achieved by turning the wheat bunch through $90^{\circ}$ after each measurement, and the lodging angles were changed manually after measurements of all lodging directions for a certain lodging angle. The measurement was conducted twice for each lodging condition.

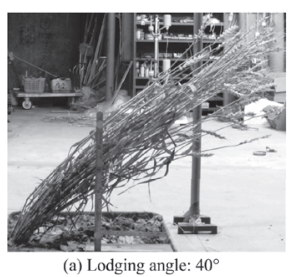

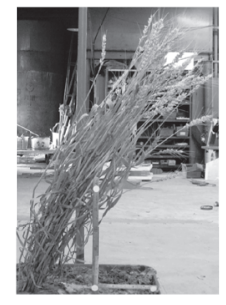

(b) Lodging angle: $62^{\circ}$

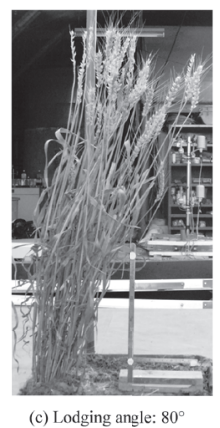

(c) Lodging angle: $80^{\circ}$
Fig. 3. Digital images of the wheat bunch in the lodging direction of "right".
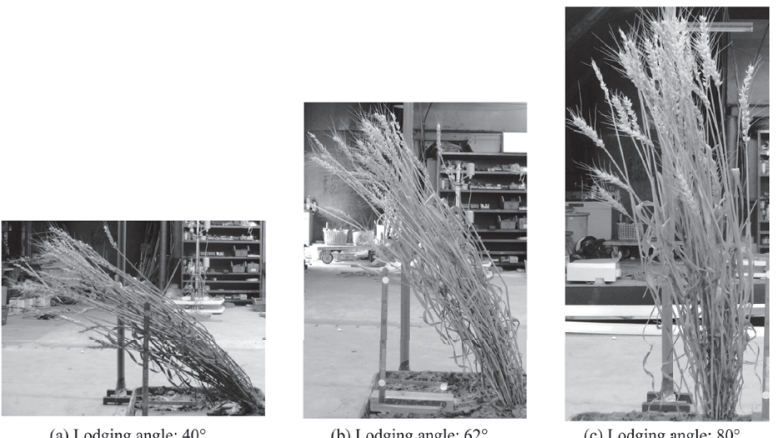

Fig. 4. Digital images of the wheat bunch in the lodging direction of "left".
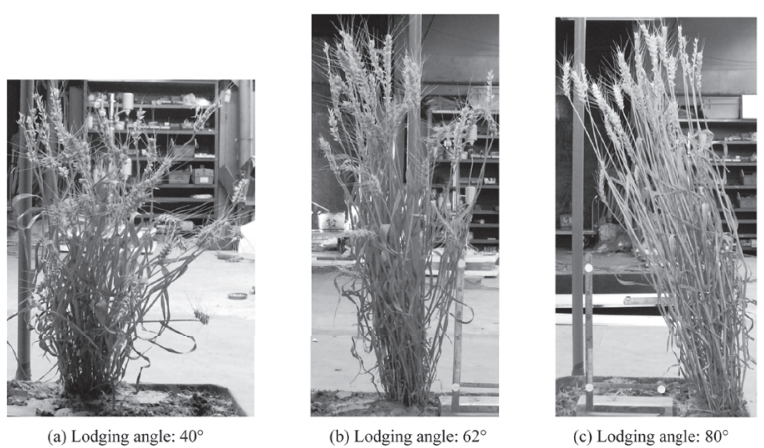

Fig. 5. Digital images of the wheat bunch in the lodging direction of "front".

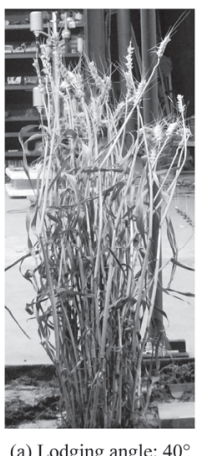

(a) Lodging angle: $40^{\circ}$

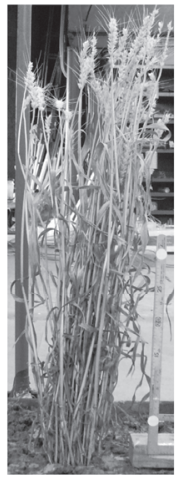

(b) Lodging angle: $62^{\circ}$

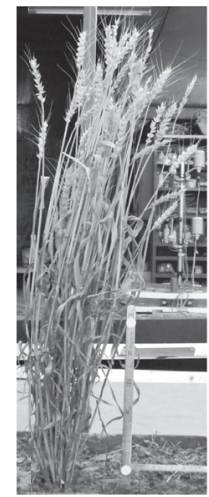

(c) Lodging angle: $80^{\circ}$
Fig. 6. Digital images of the wheat bunch in the lodging direction of "back".

The forward speed of the sensor was approximately $25 \mathrm{~mm} / \mathrm{sec}$. The rotational angle of the laser scanner $\theta$ was set in $-34^{\circ}$ against $y$-axis in the counterclockwise directions for all lodging directions in the lodging angle of $80^{\circ}$, and front and left lodging directions in the lodging angle of $62^{\circ}$. For the other conditions, the rotational angle of the sensor was set in $-53^{\circ}$.

\section{EXPERIMENTAL RESULTS AND DISCUSION}

Partial posture profiles of a wheat bunch measured by the laser scanner are shown in Fig. 7, Fig. 8, Fig. 9, and Fig. 10. Figure 7(a) is a top view for the wheat bunch which is inclined in the positive $x$ direction. The 
figure showed that the profiles extended in the $x$ direction while the extent of lodging increased (lodging angles decreased). In a front view shown in Fig. 7(b), it was judged through the comparisons with the digital images (Fig. 4) that inclination of the wheat bunch was well detected for lodging angles of $40^{\circ}$ and $62^{\circ}$. On the other hand, only upper profile of the wheat bunch was measured for the lodging angle of $80^{\circ}$, which was in an upright condition. Thus, the inclination was not detected well. For the lodging direction of "left", the same trend was confirmed, but only upper profile was measured for the lodging angle of $62^{\circ}$, too, due to the inappropriate setting for an angle of the sensor. For the lodging direction of "front" (Fig. 9), a top view showed that the profiles extended in the $y$ direction while the extent of lodging increased (lodging angles decreased).

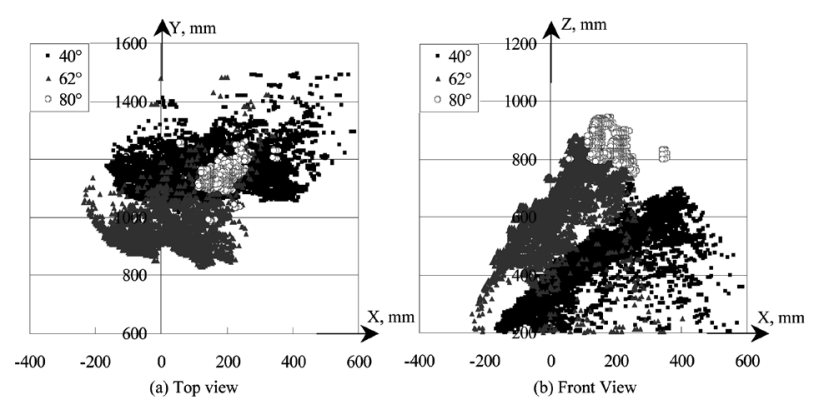

Fig. 7. Partial posture profiles of the wheat bunch in the lodging direction of "right".

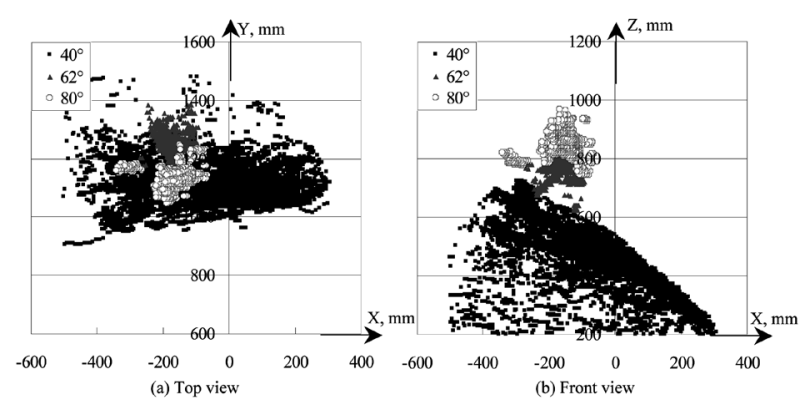

Fig. 8. Partial posture profiles of the wheat bunch in the lodging direction of "left".

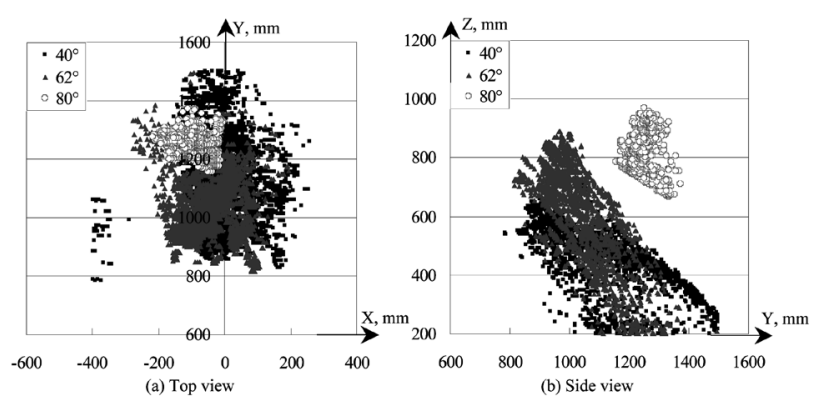

Fig. 9. Partial posture profiles of the wheat bunch in the lodging direction of "front".

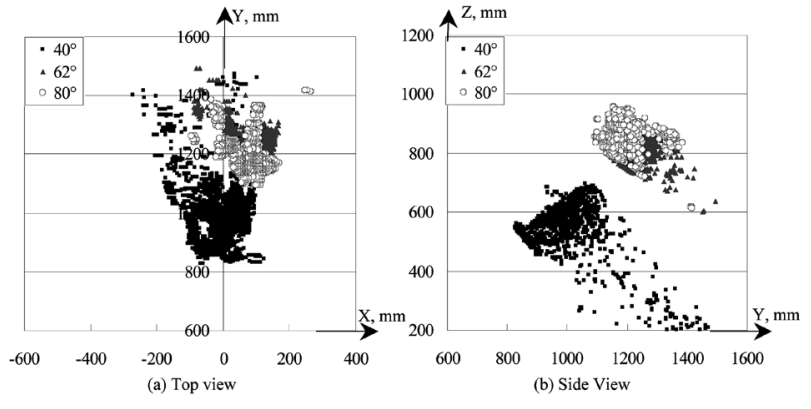

Fig. 10. Partial posture profiles of the wheat bunch in the lodging direction of "back"

Figure 9(b) showed that inclination of the wheat bunch was well detected except for an upright condition. For the lodging direction of "back", profiles around the base of the wheat bunch was not detected well because the sensor was not directed downward enough.

Next, inclination, skewness, and kurtosis were calculated based on three dimensional coordinates measured by the laser scanner, in order to assess the effectiveness of those values as indications representing lodging characteristics. The values of inclination in the $x, y$ directions were calculated by the planar approximation. Skewness and kurtosis were calculated by equation (6) and equation (7), respectively.

$$
\begin{aligned}
& S k=\frac{1}{n} \sum_{i=1}^{n}\left(d_{i}-\bar{d}\right)^{3} / s^{3} \\
& K u=\frac{1}{n} \sum_{i=1}^{n}\left(d_{i}-\bar{d}\right)^{4} / s^{4}-3
\end{aligned}
$$

where, $d_{i}$ : coordinate data of an arbitrary point measured, $\bar{d}$ : average of coordinate data used in the calculation, $i$ : index, $K u$ : kurtosis, $n$ : total number of coordinates used in the calculation, $s$ : standard deviation, $S k$ : skewness. Skewness is the indication that represents the extent of symmetry for data distribution. A positive value of skewness indicates that the peak of the data distribution is distorted to the left comparing with a normal distribution. Also, a negative value means that the peak of the data distribution is distorted to the right. Kurtosis is the indication that represents how much the data distribution is away from a normal distribution. A negative large value of kurtosis indicates that the distribution varies widely. On the other hand, a positive large value means that the distribution has a sharp peak comparing with a normal distribution. The results are shown in table 2. In the table, the combination of an alphabet and number such as "R40" expresses lodging conditions. $\mathrm{R}$ and 40 of "R40" represents the initial letter of the lodging direction (right) and a lodging angle, respectively. Also, in inclination in table 2 , positive values mean angles measured from the positive axes, and negative values mean angles measured from the negative axes. The values of inclination for R40, R62, L40, F40, and F62 were roughly agreed with actual lodging angles. The reason for errors in the values of inclination was examined 
Table 2. Inclination, skewness, and kurtosis

\begin{tabular}{|c|c|c|c|c|c|c|c|c|}
\hline & \multicolumn{2}{|c|}{ Inclination, degree } & \multicolumn{3}{|c|}{ Skewness } & \multicolumn{3}{|c|}{ Kurtosis } \\
\hline & $\mathrm{X}$ & $\mathrm{Y}$ & $\mathrm{X}$ & $\mathrm{Y}$ & $\mathrm{Z}$ & $\mathrm{X}$ & $\mathrm{Y}$ & $\mathrm{Z}$ \\
\hline $\mathrm{R} 40$ & 32.8 & -11.4 & 0.1 & 1.1 & -0.2 & -0.9 & 1.5 & -0.8 \\
\hline R62 & 44.8 & -34.2 & 0.0 & 1.9 & -0.4 & -0.6 & 5.4 & -0.4 \\
\hline $\mathrm{R} 80$ & -24.0 & -12.3 & 0.5 & 0.0 & 0.1 & 1.2 & 1.9 & -0.9 \\
\hline L40 & -24.5 & -0.5 & 0.0 & 0.6 & -0.1 & -0.9 & 1.6 & -0.7 \\
\hline L62 & -10.9 & 1.2 & 0.0 & 0.5 & -0.2 & -0.4 & 0.4 & 0.0 \\
\hline L80 & 11.9 & -25.2 & -1.4 & 0.4 & 0.0 & 1.8 & 1.0 & -0.7 \\
\hline F40 & -13.2 & -24.7 & -0.4 & 0.3 & -0.5 & 3.5 & -0.7 & -0.2 \\
\hline F62 & -27.3 & -49.3 & 0.1 & 0.6 & -0.6 & -0.2 & 0.2 & -0.4 \\
\hline F80 & -16.9 & 3.9 & -0.3 & 0.4 & 0.1 & -0.6 & 0.3 & -0.7 \\
\hline B40 & 14.9 & -14.0 & -0.7 & 1.3 & -1.7 & 0.1 & 3.2 & 3.9 \\
\hline B62 & 31.6 & 22.1 & -1.3 & 1.6 & -0.2 & 1.9 & 5.0 & 0.1 \\
\hline B80 & 0.8 & -5.3 & -0.1 & 1.5 & -0.3 & 0.5 & 2.2 & -0.1 \\
\hline
\end{tabular}

because the inclination was calculated by the planar approximation although the wheat bunch was bent with some curvature. The reason that inclination was not calculated well under the other conditions was examined that only upper profiles of lodging posture were measured due to an upright condition and the inappropriate setting for an angle of the sensor. Because values of skewness in the $y$ direction were relatively large for R40 and R60, it was examined that density of a wheat stalk was large below the average on $y$ coordinates of the posture profile. The same trend was shown in B40, B62, and B80, but it was caused by partial posture profiles inappropriately detected due to the setting for an angle of the sensor. For the values of kurtosis, information on lodging directions can be obtained. The case of small number in the $x$ direction and relatively large positive number in the $y$ direction, such as R40, R62, and L40, is judged that the wheat bunch is inclined in the $x$ direction. The case of large number in the $x$ direction and small number in the $y$ direction, such as F40, is judged that the wheat bunch is inclined in the $y$ direction. Large numbers in both of the $x$ and $y$ directions, such as R80 and L80 can be indication that the wheat bunch is in an upright condition. The large number of B40 in the $z$ direction shows that only upper portion of the wheat bunch was measured due to the inappropriate setting of the sensor.

\section{CONCLUSIONS}

A real time sensing technique for detecting lodging posture of crops was investigated using a laser scanner. The following conclusions were drawn from this study
1) Inclination of a wheat bunch was roughly detected for relatively large lodging angles. On the other hand, because only upper profiles of a wheat bunch were measured under an upright condition, the inclination was not detected well.

2) Indications such as inclination, skewness, and kurtosis can provide useful information for lodging characteristics.

3) The laser scanner applied in this study showed useful performance under certain conditions, but practical use is considered to be difficult because the performance sensitively depends on the sensor setting for an angle against objects detected.

\section{ACKNOWLEDGEMENTS}

This research was supported by a Grant-in-Aid Scientific Research (No. 19780188) from the Ministry of Education, Culture, Sports, Science and Technology, Japan.

\section{REFERENCES}

Fox, F. A. and L. E. Wagner. 2001 A laser distance-based method for measuring standing residue. Proceedings of International Symposium in Soil Erosion Research for the $21^{\text {st }}$ Century, 206-209

Monta, M., K. Namba and N. Kondo. 2004 Three dimensional sensing using laser scanner. Proceedings of ASAE/CESA Annual International meeting. ASAE Paper No. 041158

Tumbo, S. D., M. Salyani, J. D. Whitney, T. A. Wheaton and W. M. Miller 2002 Investigation of laser and ultrasonic ranging sensors for measurements of citrus canopy volume. Applied Engineering in Agriculture, 18(3): 367-372 